\title{
What is the truth about renin angiotensin blockers for diabetic patients?
}

\author{
Atsuko Abiko ${ }^{1}$ (]
}

Received: 13 July 2016/Published online: 31 August 2016

(C) The Japan Diabetes Society 2016

Keywords Diabetic nephropathy · RAS blocker . Antihypertensive agent · Meta-analysis · End-stage renal disease

Many type 2 diabetic patients have concomitant hypertension. These patients require treatment to maintain their blood pressure (BP) within acceptable ranges to decrease the risk of cardiovascular disease or diabetic nephropathy. Lowering BP can decrease the risk or slow the progression of diabetic nephropathy, and thus it is important to evaluate diabetic patients with albuminuria and perform proper staging of diabetic nephropathy. In the Action in Diabetes and Vascular disease: preterAx and diamicroN-MR Controlled Evaluation (ADVANCE) study in 2009 [1], of 11,140 patients with type 2 diabetes, those who achieved lower BP presented fewer renal events.

The BP target for hypertensive patients with diabetes is $<130 / 80 \mathrm{mmHg}$ in Japan. The Guidelines of the Japan Diabetes Society and the Japan Hypertension Society recommend angiotensin-converting enzyme (ACE) inhibitors or angiotensin receptor blockers (ARBs) as the first choice of antihypertensive treatment for diabetic patients $[2,3]$. As recommended, we usually prescribe a renin angiotensin system (RAS) blocker to diabetic patients with hypertension. However, the treatment goal of BP for patients with diabetes and hypertension is $<140 / 90 \mathrm{mmHg}$ based on the recommendations from the American Diabetes Association

Atsuko Abiko

aabiko@asahikawa-med.ac.jp

1 Division of Metabolism and Biosystemic Science, Department of Medicine, Asahikawa Medical University, Midorigaoka-Higashi 2-1-1-1, Asahikawa, Hokkaido 078-8510, Japan
[4]. The evidence supporting this goal was obtained from the Action to Control Cardiovascular Risk in Diabetes (ACCORD) trial. In that trial, no benefit was found regarding cardiovascular outcomes when comparing aggressive BP treatment goals $(<120 \mathrm{mmHg})$ with moderate treatment goals $(<140 \mathrm{mmHg})$ in patients with type 2 diabetes [5]. The ONgoing Telmisartan Alone and in combination with Ramipril Global Endpoint Trial (ONTARGET) also assessed the BP levels at which cardiovascular protection is achieved with treatment by ACE inhibitors, ARB, or both for diabetic and nondiabetic patients [6]. In that subgroup analysis, a progressive reduction in the incidence of stroke was observed when systolic BP reached $115 \mathrm{mmHg}$, but a J-curve relationship was observed for cardiovascular death and myocardial infarction. In 2015, Edmin and colleagues reported a systematic review and meta-analysis on the association between BP-lowering treatment and vascular outcomes (including macrovascular and microvascular outcomes) in type 2 diabetes [7]. They reported that each $10 \mathrm{mmHg}$ decrease in systolic BP was significantly associated with improved mortality, cardiovascular disease, coronary heart disease, stroke, retinopathy, and albuminuria. They also evaluated outcomes stratified by achieved systolic BP. The outcomes of stroke and albuminuria were also significantly reduced in the stratum with $\mathrm{BP}$ lower than $130 \mathrm{mmHg}$.

In a recent meta-analysis published in the $B M J$, Bangalore and colleagues evaluated the outcomes of diabetic patients treated with RAS blockers compared with other antihypertensive agents [8]. They evaluated 19 randomized controlled trials that enrolled 25,414 diabetic patients and were followed up for a mean of 3.8 years. The trials included in that meta-analyses were published between 1998 and 2012, and most of the trials began before 2000 . In 2003, the Seventh Report of the Joint National 
Committee on the Prevention, Detection, Evaluation, and Treatment of High Blood Pressure (JNC 7) proposed that the goal of BP for diabetic patients should be less than 130/80 mmHg [9]. The results of the meta-analysis by Bangalore et al. were surprising because RAS blockers were associated with similar risk of death (including cardiovascular death), myocardial infarction, angina pectoris, stroke, heart failure, revascularization, and endstage renal disease compared with other antihypertensive agents in diabetic patients. The summary of each relative risk of the outcomes is shown in Table 1. Many studies on RAS blockers have reported that these agents can reduce albuminuria and also suppresses progression of diabetic nephropathy; therefore, the results of this meta-analysis were unexpected. Bangalore et al. chose trials that compared RAS blockers with calcium channel blockers (15 trials), thiazide diuretics (three trials), and $\beta$-blockers (two trials). They found only a few trials comparing RAS blockers with diuretics or $\beta$-blockers, which was one of the limitations of this analysis. Regarding RAS blockers versus calcium channel blockers, RAS blockers were associated with a significant reduction in the risk of heart failure, but there were no differences in other outcomes (Table 1). The Antihypertensive and Lipid-Lowering Treatment to Prevent Heaert Attack Trial (ALLHAT) had the largest sample size in this meta-analysis and also failed to show the superiority of RAS blockers compared with other antihypertensive agents in its results [10]. ALLHAT might have a strong effect on the results of this meta-analysis, and this is one of the limitations of such meta-analyses. One trial included in this meta-analysis is the Irbesartan Type 2 Diabetes Nephropathy Trial (IDNT), which showed the superiority of a RAS blocker (irbesartan) over a calcium channel blocker (amlodipine) in 2001 [11]. This study showed that the irbesartan group was associated with a significant reduction in the risk of doubling of serum creatinine levels and end-stage renal disease compared with the amlodipine or placebo group. Further, the relative risk of the primary end point in the placebo and amlodipine groups did not differ significantly.

In contrast, Palmer et al. reported the comparative efficacy and safety of pharmacological agents aimed at lowering BP in adults with diabetes and kidney disease [12]. They analyzed 157 studies comprising 43,256 participants, mostly with type 2 diabetes and chronic kidney disease by network meta-analysis. The network meta-analysis employed was a very unique method for evaluating a large number of studies. In their results, no BP-lowering strategies prolonged survival in adults with diabetes and kidney disease. However, ACE inhibitors and ARBs, alone or in combination, were the most effective strategies against end-stage kidney disease. The secondary outcomes for renal function in this network meta-analysis were doubling of serum creatinine and regression of albuminuria. Bangalore's meta-analysis only evaluated end-stage kidney disease as a renal outcome. Therefore, the differences in outcomes between these meta-analyses are sizable. Another important difference between this network metaanalysis and Bangalore's meta-analysis is whether placebocontrolled studies were included or not.

In another network meta-analysis reported in 2013, Wu et al. showed that ACE inhibitors exerted renoprotective effects in diabetic patients and were superior to other treatments [13]. In that meta-analysis the outcomes for the renoprotective effect evaluation were progression to endstage renal disease or doubling of serum creatinine levels. These network meta-analyses could evaluate the effectiveness about ACE inhibitors and ARBs separately, but Bangalore et al. analyzed about 19 trials that combined 13 trials with ACE inhibitors and 6 trials with ARBs. In fact, both ACE inhibitors and ARBs reduce the effect of angiotensin II, but each works through a different mechanism and absolutely classifies a different class of antihypertensive agents.

A serious issue with that meta-analysis is the difference of endpoints for evaluating renal disease progression in each clinical study. Development of end-stage renal disease

Table 1 Summary of each outcome with RAS blockers versus other hypertensive agents from the meta-analysis by Bagalore et al.

\begin{tabular}{lllll}
\hline & Versus other antihypertensive agents & Versus calcium channel blockers & Versus diuretics & Versus $\beta$ blockers \\
\hline All-cause mortality & $0.99(0.93-1.05)$ & $1.01(0.92-1.10)$ & $0.99(0.90-1.08)$ & $0.84(0.47-1.51)$ \\
Cardiovascular mortality & $1.02(0.83-1.24)$ & $1.17(0.90-1.50)$ & $0.50(0.05-5.46)$ & $0.87(0.47-1.60)$ \\
Myocardial infarction & $0.87(0.64-1.18)$ & $0.84(0.54-1.30)$ & $0.14(0.01-2.74)$ & $1.02(0.73-1.40)$ \\
Angina pectoris & $0.80(0.58-1.11)$ & $0.69(0.33-1.42)$ & $0.20(0.01-4.12)$ & $0.89(0.60-1.21)$ \\
Stroke & $1.04(0.92-1.17)$ & $1.08(0.90-1.28)$ & $0.98(0.69-1.38)$ & $1.19(0.50-2.83)$ \\
Heart failure & $0.90(0.76-1.07)$ & $0.78(0.70-0.88)$ & $1.11(0.93-1.32)$ & $1.19(0.50-2.83)$ \\
Revascularization & $0.97(0.77-1.22)$ & $1.01(0.74-1.39)$ & $1.18(0.93-1.50)$ & $0.90(0.22-3.58)$ \\
End-stage renal disease & $0.99(0.78-1.28)$ & $0.88(0.64-1.21)$ & & $0.92(0.65-1.30)$
\end{tabular}

Relative risk (95\% confidence interval) 
(needed for dialysis or kidney transplantation) is considered a hard renal endpoint. Doubling of serum creatinine levels is considered a stringent renal endpoint. Beyond these outcomes, change in 24-h creatinine clearance and progression or regression of albuminuria are other parameters to assess renal function. In fact, the estimated glomerular filtration rate (eGFR) may not be reliable because of ethnic differences. The Modification of Diet in Renal Disease (MDRD) Study equation was most frequently used before 2009, and the Chronic Kidney Disease Epidemiology Collaboration (CKD-EPI) has been a major equation recently. The CKD-EPI has been modified according to several races or ethnicities by reason of the difference in creatinine generation due to muscle mass or diet pattern. Several kinds of eGFR could be mixed in the meta-analysis, so we need to pay attention to understand such a meta-analysis. If we choose a hard renal endpoint for verification of the effectiveness of antihypertensive agents, we need long follow-up periods to get a sufficient number of endpoint results. There are several arguments as to which renal endpoint is better for clinical trials aimed to evaluate the effectiveness of a therapeutic approach. In 2014, prior to the joint National Kidney Foundation (NKF)/ US Food and Drug Administration (FDA) workshop, they confirmed decline in GFR of 40 or $30 \%$ could be used as an endpoint in clinical trials of CKD [14].

Lowering of BP with pharmacological agents has been central to the treatment of diabetic kidney disease for decades, and it has been credited with the decreased prevalence of end-stage kidney disease over the past 10 years in the USA [15]. In Japan, the leading indication for initiating hemodialysis since 1998 is diabetic nephropathy. The peak ratio in 2009 was $44.5 \%$, and it has not increased further in the last 5 years [16]. It is considered that the combined use of antihypertensive agents, including RAS blockers, is a large contributor to the prevention of end-stage renal disease in the twenty-first century in spite of the growing number of diabetic patients year by year. It may be difficult to start new head-to-head trials with RAS blockers and other antihypertensive agents for diabetic patients in the future. Therefore, we should carefully consider how to choose antihypertensive agents based on the evidence from recent meta-analyses such as that by Bangalore et al. [8]. Currently, seven ARBs and 12 ACE inhibitors are available in Japan. Additionally, there are many combined preparations of ARBs and Ca blockers or diuretics. I consider that we have been prescribing these types of antihypertensive medications to diabetic patients for some time. The number of older patients with diabetes is also rising. These patients are at a particular risk of polypharmacy. Thus, it is important to consider that perhaps strict BP control may not be necessary to maintain their quality of life and function in terms of activities of daily living. In addition, excess antihypertensive treatment can cause adverse effects related to hypotension in older patients, especially those who have advanced atherosclerotic lesions. Therefore, we need to avoid hypotension for such patients. For daily clinical practice, we should be knowledgeable about the characteristics of each type of antihypertensive agent available in our setting. Further, we should repeatedly and carefully assess our diabetic patients for expected adverse effects and long-term efficacy.

\section{Compliance with ethical standards}

Ethics policy This article does not contain any studies with human or animal subjects performed by the author.

Conflict of interest The author declares that there is no conflict of interest.

\section{References}

1. de Galan BE, Perkovic V, Ninomiya T, Pillai A, Patel A, Cass A, Neal B, Poulter N, Harrap S, Mogensen CE, Cooper M, Marre M, Williams B, Hamet P, Mancia G, Woodward M, Glasziou P, Grobbee DE, MacMahon S, Chalmers J, ADVANCE Collaborative Group. Lowering blood pressure reduces renal events in type 2 diabetes. J Am Soc Nephrol. 2009;20:883-92.

2. The Japanese Diabetes Society. Guideline for the Treatment for Diabetes in Japan 2016. Nankodo Co., Ltd., Tokyo; 2016 (Japanese).

3. The Japanese Society of Hypertension. Guideline for the management of hypertension 2014. Life Science Publishing Co., Ltd., Tokyo; 2014 (Japanese).

4. American Diabetes Association. Cardiovascular disease and risk management. Diabetes Care. 2016;39(Suppl. 1):S60-71.

5. ACCORD Study Group. Cushman WC, Evans GW, Byington RP, Goff DC Jr, Grimm RH Jr, Cutler JA, Simons-Morton DG, Basile JN, Corson MA, Probstfield JL, Katz L, Peterson KA, Friedewald WT, Buse JB, Bigger JT, Gerstein HC, Ismail-Beigi F. Effects of intensive blood-pressure control in type 2 diabetes mellitus. N Engl J Med. 2010; 362:1575-85.

6. Redon J, Mancia G, Sleight P, Schumacher H, Gao P, Pogue J, Fagard R, Verdecchia P, Weber M, Böhm M, Williams B, Yusoff K, Teo K. Yusuf S; ONTARGET Investigators. Safety and efficacy of low blood pressures among patients with diabetes: subgroup analyses from the ONTARGET (ONgoing Telmisartan Alone and in combination with Ramipril Global Endpoint Trial). J Am Coll Cardiol. 2012;59:74-83.

7. Edmin CA, Rahimi K, Neal B, Callender T, Perkovic V, Patel A. Blood pressure lowering in type 2 diabetes A systematic review and meta-analysis. JAMA. 2015;313:603-15.

8. Bangalore S, fakheri R, Toklu B, messerli FH. Diabetes mellitus as a compelling indication for use of renin angiotensin system blockers: systemic review and meta-analysis of randomizes trials. BMJ. 2016; 352:i438. doi:10.1136/bmj.i438.

9. Chobanian AV, Bakris GL, Black HR, Cushman WC, Green LA, Izzo JL Jr, Jones DW, Materson BJ, Oparil S, Wright JT Jr, Roccella EJ, National Heart, Lung, and Blood Institute Joint National Committee on Prevention, Detection, Evaluation, and Treatment of High Blood Pressure; National High Blood Pressure Education Program Coordinating Committee. The seventh report of the joint national committee on prevention, detection, 
evaluation, and treatment of high blood pressure: the JNC 7 report. JAMA. 2003;289:2560-72.

10. Whelton PK, Barzilay J, Cushman WC, Davis BR, Iiamathi E, Kostis JB, Leenen FH, Louis GT, Margolis KL, Mathis DE, Moloo J, Nwachuku C, Panebianco D, Parish DC, Pressel S, Simmons DL, Thadani U, ALLHAT Collaborative Research Group. Clinical outcomes in antihypertensive treatment of type 2 diabetes, impaired fasting glucose concentration, and normoglycemia: antihypertensive and lipid-lowering treatment to prevent heart attack trial (ALLHAT). Arch Intern Med. 2005;165:1401-9.

11. Lewis EJ, Hunsicker LG, Clarke WR, Berl T, Pohl MA, Lewis JB, Ritz E, Atkins RC, Rohde R, Raz I, Collaborative Study Group. Renoprotective effect of the angiotensin-receptor antagonist irbesartan in patients with nephropathy due to type 2 diabetes. N Engl J Med. 2001;345:851-60.

12. Palmer SC, Mavridis D, Navarese E, Craig JC, Tonelli M, Salanti G, Wiebe N, Ruospo M, Wheeler DC, Strippoli GF. Comparative efficacy and safety of blood pressure-lowering agents in adults with diabetes and kidney disease: a network meta-analysis. Lancet. 2015;385:2047-56.

13. Wu H-Y, Huang J-W, Lin H-J, Liao WC, Peng YS, Hung KY, $\mathrm{Wu} \mathrm{KD}, \mathrm{Tu} \mathrm{YK}$, Chien KL. Comparative effectiveness of reninangiotensin system blockers and other antihypertensive drugs in patients with diabetes: systematic review and Bayesian network meta-analysis. BMJ. 2013;347:f6008.

14. Levey AS, Inker LA, Matsushita K, Greene T, Willis K, Lewis E, de Zeeuw D, Cheung AK, Coresh J. GFR decline as an end point for clinical trials in CKD: a scientific workshop sponsored by the National Kidney Foundation and the US Food and Drug Administration. Am J Kidney Dis. 2014;64:821-35.

15. Burrows NR, Li Y, Geiss LS. Incidence of treatment for endstage renal disease among individuals with diabetes in the US continues to decline. Diabetes Care. 2010;33:73-7.

16. Japanese Society for Dialysis Therapy. An overview of regular dialysis treatment in Japan as of Dec. 31, 2014 (Japanese). http:// docs.jsdt.or.jp/overview/index.html. 